\title{
Efecto de la micorrización y el lombriabono sobre el crecimiento y desarrollo del Sacha inchi Plukenetia volubilis $\mathrm{L}$.
}

\section{Effect of mycorrhizal and lombriabono on growth and development of Sacha inchi Plukenetia volubilis L}

\author{
Luís Alejandro Pérez Caro', Luís Eliecer Oviedo Zumaqué2*, José Luís Barrera Violeth³
}

Recibido para publicación: Mayo 24 de 2017 - Aceptado para publicación: Noviembre 23 de 2017

\begin{abstract}
RESUMEN
El sacha inchi Plukenetia volubilis es una Euphorbiaceae de importancia socioeconómica debido al alto valor nutricional de su semilla; siendo importante establecer estrategias agronómicas que incremente los rendimientos; por lo cual se evaluó el efecto de la micorrización y del lombriabono, en el crecimiento y desarrollo de la planta. La investigación se realizó en el corregimiento de Berástegui, municipio de Ciénaga de oro, Córdoba, bajo un diseño de bloques al azar con tres repeticiones y los tratamientos T1: Control, sin aplicación, T2: $100 \mathrm{gr} /$ planta de Micorriza comercial (micorrizafer) y T3: 2 kg/ planta con lombriabono. Los tratamientos se aplicaron a los 45 días después de siembra (DDS). Las evaluaciones se efectuaron de acuerdo al ciclo fenológico (crecimiento vegetativo y reproductivo) del cultivo, cuantificando la altura de planta y diámetro del tallo a los 52, 97 y 136 DDS, número de flores femeninas; número de flores fecundadas y número de flores masculinas 112, 128, 144 y 168 DDS y el número de capsulas 128, 144, 168 y 176 DDS. Las variables evaluadas en plantas inoculadas con micorrizas fueron significativamente mayor $(\mathrm{P}<0,05)$ que cuando se fertilizó con lombriabono superándolo en altura $(0,23 \mathrm{~m})$, diámetro del tallo en $(0,08 \mathrm{~cm})$, número de flores femeninas (8 flores), número de flores fecundadas (38 flores) y número flores masculinas (18 flores). Se concluye, que la planta de Sacha inchi, es dependiente de la micorrización y responde significativamente a planes de fertilización cuando se incluye micorrizas.
\end{abstract}

Palabras clave: Plukenetia volubilis L., bioinsumo, lombriabono, micorriza.

\begin{abstract}
The sacha inchi Plukenetia volubilis is an Euphorbiaceae of socioeconomic importance due to the high nutritional value of its seeds. Therefore, it is important to establish agronomic strategies that increase economic yields. This study evaluated the effects of mycorrhizal and vernicompost on the growth and development of the plant. The investigation was carried out in the village of Berástegui, municipality of Ciénaga de Oro, Córdoba, under a randomized block design with three replicates and treatments T1: Control, without application, T2: 100 gr/plant of commercial mycorrhiza (micorrizafer) and T3: $2 \mathrm{~kg} /$ plant whit vernicompost. The treatments were applied at 45 days after sowing (DDS). The evaluations were carried out according to the phenological cycle (vegetative and reproductive) of the culture, quantifying the plant height and stem diameter at 52 -97-136 DDS, number of female flowers, number of fecund flowers and number of male flowers $112-128$ - 144 - 168 DDS and number of Capsules 128-144-167-176 DDS. The variables evaluated in plants inoculated with mycorrhizal were significantly higher $(\mathrm{P}<0.05)$ than when fertilized with vernicompost exceeding in height $(0.23 \mathrm{~m})$, stem diameter in $(0.08 \mathrm{~cm})$, number of female flowers ( 8 flowers), number of fertilized flowers (38 flowers) and number of male flowers (18 flowers). It is concluded that the Sacha inchi plant is dependent on mycorrhization and responds significantly to fertilization plans when mycorrhizae are included.
\end{abstract}

Key words: Plukenetia volubilis L., biosupplies, vernicompost, mycorrhizae.

\footnotetext{
1 Biólogo, biodecluisalejandro@gmail.com. Calle $8^{a}$ No 22 - 46. Ciénaga de Oro, Colombia

2* M.Sc. Docente Universidad de Córdoba, Departamento de Biología. Carrera 6 No. 76-103, Montería, Colombia. Teléfono (4) 7860589, telefax (4) 7909429. luisoviedo59@gmail.com,

3 M.Sc. Docente Universidad de Córdoba, Departamento de Ingeniería Agronómica y Desarrollo Rural. Carrera 6 No. 76-103, Montería, Colombia. jlbarrera@correo.unicordoba.edu.co
} 
Pérez et al. - Nutrición orgánica de Plukenetia volubilis L.

\section{INTRODUCCIÓN}

Plukenetia volubilis es una especie de la familia Euphorbiaceae, originaria de la amazonia peruana, zona tropical húmeda, presenta importancia socioeconómica en razón del alto valor nutricional de sus semillas, las cuales contienen $48,6 \%$ de lípidos polinsaturados esenciales (linoleico y linolénico) y $29 \%$ de proteína (Arévalo, 1999), y al gran potencial agroexportador, que actualmente lo están efectuando países como Ecuador, Bolivia, Chile.

En Colombia se cultiva en los departamentos de Putumayo, Caquetá, Caldas y Antioquia (Peña, 2008 y Espinoza et al., 2009).

Según AGRONET (2015), los mayores productores de $P$. volubilis son Putumayo con un área de 33 hectáreas y Chocó con una hectárea, reportando rendimientos de 1,65 y 0,5 toneladas de semilla por hectárea. El contenido total de aceite presente en la semilla que se produce por departamento es $41,4 \%$ y $30 \%$ respectivamente.

La práctica de la fertilización como medio para mejorar el rendimiento de los cultivos, tiene sus orígenes en los inicios de la agricultura, y desde entonces, se ha venido mejorando sustancialmente. Sin embargo, el gran salto que se ha dado en el manejo de los fertilizantes, su función en el metabolismo y su impacto en la producción de los cultivos, comienza a partir de 1880 con los descubrimientos de las funciones de diferentes elementos en la fisiología vegetal. Los nuevos conocimientos generados, sentaron las bases para la utilización creciente de fertilizantes orgánicos constituidos por N, P y $\mathrm{K}$, (como es el caso del lombriabono) con la finalidad de inducir mayores rendimientos en cultivos agrícolas (Finck, 1988).

En los últimos años, se ha empezado a utilizar hongos micorrizicos arbusculares, para mejorar la respuesta agronómica de los cultivos, lo que ha adquirido gran importancia en el ámbito científico (Medina et al., 2000; Baar, 2008).

Estos organismos además, de incrementar la producción, favorecen la resistencia al estrés hídrico (Al-Karaki et al., 2004; Cardoso y Kuyper, 2006), incrementan la producción de clorofila (Ekanayake et al., 2004) y ejercen influencia sobre la resistencia de las plantas y sobre su capacidaddesobrevivirencondicionesadversas, lo que ha sido fundamentado, por diferentes autores donde corroboran que las micorrizas incrementan en las plantas la capacidad de absorción de nutrientes (Purakayastha y Chhonkar, 2005; Karandashov y Bucher, 2005).

Sacha inchi es una especie empleada en la industria alimentaria, fotoquímica, farmacéutica y cosmecéutica (Yance, 2009) y a pesar de que el cultivo de esta oleaginosa viene siendo promovido por su fácil adaptación y su elevada capacidad de producción de aceites, en el departamento de Córdoba son pocos los estudios que se han realizado con respecto al efecto de la micorrización y el abonamiento orgánico en el desarrollo fisiológico y el rendimiento de la especie.

Debido a esta falta de estudios sobre estrategias de manejo sostenible del cultivo, en el departamento se desconoce el potencial productivo de esta especie, así como la trascendencia económica e industrial que ha venido tomando en los últimos años en el mercado nacional e internacional como consecuencia de la demanda ascendente de ácidos grasos poliinsaturados.

Dada la importancia quetieneel establecimiento de planes de micorrización y abonamiento que permitan mayor aprovechamiento de nutrientes en sacha inchi, se planteó como objetivo evaluar la respuesta de la especie Plukenetia volubilis L. la aplicación de bioinsumo lombriabono y hongos micorrizicos vesículo arbusculares, en el municipio de Ciénaga de oro, Córdoba. 


\section{METODOLOGÍA}

La investigación se realizó en el corregimiento de Berástegui ubicado a $9 \mathrm{Km}$ de la cabecera del Municipio de Ciénaga de Oro; localizado geográficamente a $8^{\circ} 52^{\prime} 41^{\prime \prime}$ de Latitud Norte y $75^{\circ} 37^{\prime} 27^{\prime \prime}$ Longitud Oeste del Meridiano de Greenwich; nororiente del departamento de Córdoba, temperatura promedio de $27,8{ }^{\circ} \mathrm{C}$, humedad relativa de $83 \%$, precipitación promedio anual de 1500 mm. Según escala de Holdrige, predomina el clima seco tropical, posee suelos de tipo entisoles e inceptisoles y presenta una altura máxima de 25 msnmm (Palencia et al., 2006).

El manejo agronómico de las unidades experimentales en cuanto a fertilización se basó en los resultados de los análisis de suelos., Las propiedades químicas arrojadas por análisis del suelo realizado en el Laboratorio de Suelos y Aguas de la Universidad de Córdoba fueron $\mathrm{pH}$ de 6,7 ; contenido de materia orgánica $(1,33 \%)$; $\mathrm{S}\left(8,6 \mathrm{mg} \mathrm{Kg}{ }^{-1}\right) ; \mathrm{P}\left(39,6 \mathrm{mg} \mathrm{Kg}{ }^{-1}\right) ; \mathrm{Ca}(11,5$ $\left.\mathrm{cmol}^{+} / \mathrm{Kg}\right) ; \mathrm{Mg}\left(6,7 \mathrm{cmol}^{(+)} \mathrm{Kg}^{-1}\right) ; \mathrm{K}\left(0,46 \mathrm{cmol}^{(+)}\right.$ $\mathrm{Kg}-1) ; \mathrm{Na}\left(0,20 \mathrm{cmol}(+) \mathrm{Kg}^{-1}\right)$ y capacidad de intercambiocatiónicoefectivo $\left(10,9 \mathrm{cmol}^{(+)} \mathrm{Kg}^{-1}\right)$.

El terreno se preparó de manera tradicional, una arada(a20 cmdeprofundidadaproximadamente) y dos pases de rastra liviana o pulida. El sistema de soporte consistió en tutores de 2,20 m de largo distanciados a $3 \mathrm{~m}$. entre surcos y $3 \mathrm{~m}$ entre puntos de siembra. Para el tutorado se utilizó alambre galvanizado a $2 \mathrm{~m}$ de altura con respecto al suelo. El trasplante se realizó a los 45 DDS cuando las plantas tenían una altura superior a $10 \mathrm{~cm}$, grosor de tallo superior a $0,3 \mathrm{~cm}$ y libre de enfermedades. El manejo de arvenses (particularmente de gramíneas), se efectuó con deshierbes manuales semanales.

Los tratamientos fueron: Control, sin aplicación (T1); aplicación de 100 gr/ planta Micorriza comercial (micorrizafer) producto comercializado por la empresa
Natural Control (T2), inoculación de hongos micorrízicos arbusculares (HMA) constituía de inóculos certificados de Glomus fasciculatum; Scutellospora heterogama; Glomus mosseae; Glomus manihotis; Acaulospora rugosa y Entrophospora colombiana. Posee un $\mathrm{pH} \quad 5,0 \quad$ - 6,0 Humedad Gravimétrica Máxima 15\% y aplicación de 2 kg/planta lombriabono comercial compostado a partir de una mezcla 1:1:0,5\% p/p de estiércol de bovino, contenido ruminal y gallinaza (T3). Posee un pH de 7,1; materia orgánica 57,10\% y $50,1 \%$ de humedad, con una composición de N (1,75\%); P $(0,24 \%)$ y K $(0,15 \%)$ y una relación carbono/nitrógeno del 10,4\%. Para la aleatorización de los tratamientos se utilizó un diseño de bloques completos al azar (BCA), con tres tratamientos y tres repeticiones por tratamientos, cada unidad experimental estuvo constituida por 30 plantas.

Para llevar a cabo el estudio se determinaron los siguientes parámetros morfofisiológicos concordantesconelciclofenológicodelaespecie.

Altura de planta y el diámetro del tallo, se determinaron a los 52, 97 y 136 (DDS), las mediciones de la altura del tallo se realizaron entre la superficie del suelo y el ápice de la planta; el diámetro del tallo, se determinó a 3 $\mathrm{cm}$ del suelo; y el conteo del número de flores (femeninas, fecundadas y masculinas) se realizó a los 112, 128, 144 y 168 DDS y número de cápsulas 128, 144, 168 y 176 DDS, se tomó como inicio de la floración cuando las plantas de cada parcela presentaron un 50\% de emergencia de flores y cápsulas, tomando el dato promedio correspondiente a seis plantas seleccionadas al azar de la hilera central de cada tratamiento.

Se realizó un análisis de varianza; se consideró $\mathrm{P}<0,05$, como diferencia significativa, además se aplicó la prueba de comparación de medias Tukey $(\mathrm{P}<0,05)$ para las variables que mostraron significancia, se utilizó el programa estadístico SAS (SAS, 2008). 
Pérez et al. - Nutrición orgánica de Plukenetia volubilis L.

\section{RESULTADOS Y DISCUSIÓN}

Los resultados obtenidos en cuanto a la altura y el diámetro del tallo se observan en las tablas 1 y 2 , solo se presentaron diferencias estadísticas significativas entre los tratamientos hasta los primeros 97 DDS para ambas variables, cuando la plantas fueron tratadas con micorrizas (T2), sin embargo hacia el final del periodo vegetativo (136 DDS) se registraron valores de 1,84 m y 1,24 $\mathrm{cm}$ respectivamente, mayor numéricamente que los demás tratamientos. En este sentido Azcón y Barea (1992) y Gianinazzi et ál., (2010) indican que las micorrizas contribuyen positivamente en la altura y diámetro del tallo debido a que la aplicación de micorrizas vesículo arbusculares en los cultivos, mejoran la capacidad de absorción de nutrientes de las plantas y aportan los nutrientes necesarios de la planta para satisfacer las demandas.

Tabla 1. Altura de planta de la especie Plukenetia volubilis L. en los tratamientos, a diferentes días después de siembra.

\begin{tabular}{lccc}
\hline & \multicolumn{3}{c}{ Altura de planta (m) } \\
Tratamientos & $\mathbf{5 0}$ DDS & 97 DDS & $\mathbf{1 3 6}$ DDS \\
\hline Control & $0,73 \mathrm{~b}$ & $1,14 \mathrm{~b}$ & $1,66 \mathrm{a}$ \\
Micorriza & $0,93 \mathrm{a}$ & $1,38 \mathrm{a}$ & $1,84 \mathrm{a}$ \\
Lombriabono & $0,49 \mathrm{c}$ & $1,09 \mathrm{~b}$ & $1,61 \mathrm{a}$ \\
\hline
\end{tabular}

*Promedios con la misma letra son estadísticamente iguales, según la prueba de Tukey al $5 \%$ de probabilidad.

La baja respuesta de las plantas de Sacha inchi a la aplicación de lombriabono en los primeros 97 DDS, se debió a la liberación lenta y gradual de nutrientes por el lombriabono a través del proceso de mineralización con el fin de mantener los niveles óptimos de suelo durante períodos prolongados de tiempo (acción residual). Así mismo, algunas de las sustancias orgánicas liberadas durante la mineralización pueden actuar como quelatos que ayudan en la absorción de hierro y otros micronutrientes (Suge et al., 2011), por consiguiente, las plantas presentaron mejor respuesta al tratamiento al final del periodo de crecimiento vegetativo
(136 DDS) donde pudieron aprovechar de una manera eficiente los nutrientes liberados por el lombriabono como el nitrógeno, potasio y fósforo para llevar a cabo funciones vitales como el crecimiento, desarrollo y mantenimiento de los órganos vegetales y la regeneración de proteínas (Montaño et al., 2009) En este sentido Sandoval (2002) reportó en el cultivo papaya (Carica papaya L.), que la proporción de $60 \%$ de vermicomposta más micorrizas siempre presentó resultados superiores respecto a la variable altura de planta con relación al resto de los tratamientos. Galindo (2008) comparó el comportamiento agronómico en plantas de frijol (Vigna unguiculata (L.) Walp) bajo el manejo agronómico con micorrizas arbusculares nativas y comerciales observando que la altura promedio fue mayor en el tratamiento cepa comercial, seguido por el tratamiento cepa nativa y por último el tratamiento testigo; Ley et al., (2015) demostraron que el establecimiento de estrategias de micorrización en el cultivo de tomate (Lycopersicon esculentum Mill.) influye positivamente en crecimiento vegetal de las plantas, obteniendo plantas con mayor altura promedio.

Tabla 2. Diámetro del tallo de la especie Plukenetia volubilis L. en los tratamientos, a diferentes días después de siembra.

\begin{tabular}{lccc}
\hline \multirow{2}{*}{ Tratamientos } & \multicolumn{3}{c}{ Diámetro del tallo $(\mathbf{m})$} \\
& 50 DDS & 97 DDS & 136 DDS \\
\hline Control & $0,42 \mathrm{~b}$ & $0,72 \mathrm{a}$ & $1,21 \mathrm{a}$ \\
Micorriza & $0,55 \mathrm{a}$ & $0,79 \mathrm{a}$ & $1,24 \mathrm{a}$ \\
Lombriabono & $0,30 \mathrm{c}$ & $0,59 \mathrm{~b}$ & $1,16 \mathrm{a}$ \\
\hline
\end{tabular}

* Promedios con la misma letra son estadísticamente iguales, según la prueba de Tukey al 5\% de probabilidad.

En lo referente al diámetro del tallo, los resultados coinciden con los obtenidos por Olivera et al., (2010), quienes estudiaron diferentes alternativas de fertilización para mejorar la producción frijol (Phaseolus vulgaris L.), indicaron que la aplicación del producto Ecomic (Micorrizas arbusculares) con un suplemento de fertilizante mineral se 
obtuvo el mayor diámetro del tallo, así mismo, Barrera et al., (2011) evaluaron la respuesta de implementación de planes de abonamiento orgánico sobre el crecimiento y producción del cultivo plátano Hartón (Musa AAB), afirmaron que la aplicación de micorrizas combinado con lombriabono mostró mayor diámetro promedio del tallo; en contraste con los demás tratamientos evaluados y finalmente Ley et al., (2015) reportaron en el cultivo de tomate (Lycopersicon esculentum Mill.) que la inoculación de cepas nativas de hongos micorrizógeno arbusculares, superó al tratamiento testigo.

En cuanto a la variable número de flores femeninas, se presentaron diferencias significativas entre los tratamientos $(\mathrm{P}<0,05)$ a los primeros 128 DDS, se observó que el número de flores femeninas fue superior cuando se inóculo con micorrizas (T2). Entre los 144 y 168 DDS no existió diferencia significativa entre los tratamientos, sin embargo, el tratamiento con micorrizas (T2) registro 45 y 56 flores respectivamente (Tablaa 3). Estos resultados evidencian que las micorrizas favorecieron la floración de las plantas de Sacha inchi, debido a la contribución fisiológica de las micorrizas en mejorar el sistema radicular, la absorción de los nutrientes naturales del suelo, además contienen fitohormonas que inciden en el crecimiento y floración (Luna et al., 2016).

La baja respuesta de las plantas de Sacha inchi a la aplicación de lombriabono en los primeros 128 DDS, se debió a la liberación paulatina de los nutriente por el lombriabono, ya que contienen nutrientes en forma orgánica (Herencia et al., 2007). Por tanto, una vez aplicados al suelo, primero mediante el proceso de mineralización son transformados en compuestos inorgánicos asimilables para las plantas como es caso del nitrógeno y del fosforo orgánico que se convierten en $\mathrm{N}$ amoniacal $\left(\mathrm{NH}_{4}^{+}\right)$y principalmente en $\mathrm{N}$ nítrico $\left(\mathrm{NO}_{3}{ }^{-}\right)$ y en fosfato di (PO42-) y monobásicos $\left(\mathrm{PO}_{4}^{-}\right)$ (Antil et al., 2009), debido a lo cual, las plantas presentaron mejor respuesta al tratamiento al final del periodo de floración (168 DDS) donde pudieron aprovechar de una manera eficiente los nutrientes liberados por el lombriabono para llevar a cabo funciones vitales como el crecimiento, desarrollo y mantenimiento de los órganos vegetales y la regeneración de proteínas (Montaño et al., 2009). Con respectos al número de flores fecundadas, se encontró que hasta el día 168 DDS se presentaron diferencias significativas $(\mathrm{P}<0,05)$ entre los tratamientos, presentando mayor promedio de flores fecundadas (34 flores) las plantas tratadas con micorrizas (T2) (Tabla 4).

El comportamiento diferencial observado en el número de flores fecundadas, para los tratamientos fue debido al efecto de la micorrizas en la asimilación de fósforo por las plantas, fundamentado por diferentes autores donde corrobora que las micorrizas toman de forma más eficiente los fosfatos en el suelo a través de sus hifas que incrementan el

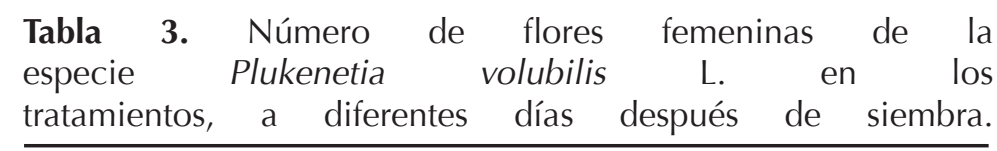

\begin{tabular}{|c|c|c|c|c|}
\hline \multirow[b]{2}{*}{ Tratamientos } & \multicolumn{4}{|c|}{ Número de flores femeninas } \\
\hline & 112 DDS & 128 DDS & 144 DDS & 168 DDS \\
\hline Control & $12 \mathrm{~b}$ & $19 b$ & $23 \mathrm{a}$ & $22 \mathrm{a}$ \\
\hline Micorriza & $33 \mathrm{a}$ & $40 \mathrm{a}$ & $45 \mathrm{a}$ & $56 \mathrm{a}$ \\
\hline Lombriabono & $11 \mathrm{~b}$ & $20 \mathrm{~b}$ & $26 \mathrm{a}$ & $26 \mathrm{a}$ \\
\hline
\end{tabular}


Pérez et al. - Nutrición orgánica de Plukenetia volubilis L.

Tabla 4. Número de flores fecundadas de la
especie
Plukenetia
tratamientos, a diferentes días después de siembra.

\begin{tabular}{|c|c|c|c|c|}
\hline \multirow[b]{2}{*}{ Tratamientos } & \multicolumn{4}{|c|}{ Número de flores fecundadas } \\
\hline & 112 DDS & 128 DDS & 144 DDS & 168 DDS \\
\hline Contro & $7 \mathrm{~b}$ & $13 \mathrm{~b}$ & $17 \mathrm{~b}$ & $18 \mathrm{~b}$ \\
\hline Micorriza & $28 \mathrm{a}$ & $33 \mathrm{a}$ & $35 \mathrm{a}$ & $34 \mathrm{a}$ \\
\hline Lombriabono & $4 \mathrm{~b}$ & $14 \mathrm{~b}$ & $17 \mathrm{~b}$ & $18 \mathrm{~b}$ \\
\hline
\end{tabular}

volumen de suelo explorado (De Prager, 2007; Cabrales et al. 2016).

La baja respuesta de las plantas de Sacha inchi a la aplicación de lombriabono en los primeros 128 DDS, se debió a la liberación lenta y gradual de nutrientes por el lombriabono a través del proceso de mineralización con el fin de mantener los niveles óptimos de suelo durante períodos prolongados de tiempo (acción residual). Por tanto las plantas presentaron mejor efecto al tratamiento al final del periodo de floración (168 DDS) donde pudieron aprovechar de una manera eficiente los nutrientes liberados por el lombriabono para llevar a cabo funciones vitales como construcción, mantenimiento, y reparación de los órganos vegetales y renovación de proteínas (Cruz et al., 2012), sin embargo, las plantas del tratamiento lombriabono continuaron mostrando menor producción de flores fecundadas respecto al tratamiento con micorrizas, puesto que, el número de flores femeninas presentes depende factores como condiciones climáticas, densidad de polen, ausencia de polinización, ya que Sacha inchi presenta un buen crecimiento y desarrollo en diversas temperaturas, sin embargo la temperatura óptima para su crecimiento, oscila con un mínimo $10{ }^{\circ} \mathrm{C}$ y un máximo de 36 ${ }^{\circ} \mathrm{C}$. Si las temperaturas son superiores por un período de tiempo prolongado puede generar caída de flores, principalmente aquellas recién formadas (Fanali et al., 2011). Además, las Iluvias provocan caída del polen, lavado de granos de polen del estigma y pérdida de viabilidad del polen (Rallo y Cuevas 2004), el aborto de flores pistiladas provocado por una alta densidad de polen en los cultivos, y/o la caída de flores por falta de polinización, Catlin y Olsson (1990), Polito et al., (1998) y Lemus (2005) señalan que ambos eventos están sujetos a la dinámica de la floración y de las condiciones ambientales que la afectan.

Referente al número flores masculinas, se presentaron diferencias significativas $(\mathrm{P}<0.05)$ en los primeros 128 DDS entre los tratamiento, presentado mayores promedios las plantas tratadas con micorrizas (T2). A los 144 DDS, los tratamientos no presentan diferencias significativas, el tratamiento con micorriza (T2), obtuvo numéricamente el mayor promedio de flores. A los 168 DDS el tratamiento con micorriza (T2) superó significativamente a los tratamientos control y lombriabono, mostrando con 63 flores mayor promedio de flores masculinas (Tabla 5).

Estos resultados demuestran que las micorrizas afecta positivamente la formación de flores, dado a que poseen propiedades que proporcionan una mayor tolerancia por parte de las plantas frente a muchos factores productores de estrés como son el déficit de humedad en el suelo, desequilibrios en el $\mathrm{pH}$, altos contenidos de sales, entre otros y también, facilita una adecuada evapotranspiración de la planta y un mejor funcionamiento fisiológico de éstas en sentido general (Parodi y Pezzani 2011). 
TEMAS AGRARIOS - Vol. 23:(1) Enero - Junio 2018 (20 - 29)

Tabla 5. Número de flores masculinas de la especie Plukenetia volubilis L. en los tratamientos, a diferentes días después de siembra.

\begin{tabular}{|c|c|c|c|c|}
\hline \multirow[b]{2}{*}{ Tratamientos } & \multicolumn{4}{|c|}{ Número de flores masculinas } \\
\hline & 112 DDS & 128 DDS & 144 DDS & 168 DDS \\
\hline Control & $19 \mathrm{~b}$ & $29 \mathrm{~b}$ & $36 a$ & $37 \mathrm{~b}$ \\
\hline Micorriza & $44 \mathrm{a}$ & $51 \mathrm{a}$ & $58 \mathrm{a}$ & $63 \mathrm{a}$ \\
\hline Lombriabono & $15 \mathrm{~b}$ & $28 \mathrm{~b}$ & $36 \mathrm{a}$ & $45 \mathrm{a}$ \\
\hline
\end{tabular}

La baja respuesta de las plantas de Sacha inchi a la aplicación de lombriabono en los primeros 128 DDS, se debió a la liberación equilibrada y distribuida de los nutrientes por el lombriabono a través del proceso de mineralización, con el fin de mantener los niveles óptimos de suelo durante períodos prolongados de tiempo (acción residual). (Suge et al., 2011), por tanto, las plantas presentaron mejor respuesta al tratamiento al final del periodo de floración (168 DDS) donde pudieron aprovechar de una manera eficiente los nutrientes liberados por el lombriabono como el N, K y P para llevar a cabo funciones vitales como el crecimiento y desarrollo de los órganos vegetales, el mantenimiento de las estructuras existentes y la regeneracióndeproteínas(AcevedoyPire,2004).

Resultados similares a los obtenidos en este estudio respecto a la floración (número de flores femeninas, fecundadas y masculinas) fueron obtenidos por Galindo (2008), al comparar el efecto de la inoculación con micorrizas vesículo arbusculares nativas y comerciales en frijol (Vigna unguiculata (L.) Walp) observó que el tratamiento cepa comercial mostró mayor promedio de flores que el tratamiento cepa nativa y el tratamiento testigo.

Díaz et al., (2013) evaluaron el impacto de la micorrización y la incorporación de composta de caña en el rendimiento de Jatropha (Jatropha curcas L.), reportando, que las plantas micorrizadas presentaron mayor promedio de flores con referencia a los tratamientos testigo y composta, confirmando la importancia del establecimiento de la simbiosis en Jatropha, que permite favorecer el transporte de nutrientes y agua a la planta y Ley et al., (2015) al inocular cepas nativas de hongos micorrizógenos arbusculares en el cultivo de tomate (Lycopersicon esculentum Mill.), observaron que en todos los períodos evaluados, los tratamientos micorrizados presentaron promedios superiores con relación al tratamiento testigo. Sin embargo, De la Rosa-mera et al., (2012) obtuvieron resultados diferentes cunado evaluaron el efecto de la inoculación con consorcios de hongos micorrízicos arbusculares en el crecimiento de vinca (Catharanthus roseus L.) reportando que no se encontraron diferencias significativas entre los tratamientos; sin embargo, los consorcios aislados de $C$. tenuiflora y $A$. capillus-veneris mostraron mayor número de flores. Mostrando que la respuesta en la formación de flores no es dependiente de la colonización micorrízica, este resultado confirma lo expresado por Daft y Nicolson (1966) quienes afirman que una asociación micorrízica no siempre incrementa el rendimiento del cultivo asociado.

En lo referente al número de cápsulas, el análisis de varianza denota que hasta 176 DDS los tratamientos no presentan diferencias significativas $(P>0,05)$, aunque el tratamiento de inoculación con micorriza (T2) con 13 cápsulas, numéricamente obtuvo el mejor promedio de cápsulas (Tabla 6). Estos resultados denotan que las micorrizas 
Pérez et al. - Nutrición orgánica de Plukenetia volubilis L.

$\begin{array}{llcccr}\text { tabla 5. Número } & \text { de } & \text { capsulas } & \text { de } & \text { la } \\ \text { especie } & \text { Plukenetia } & \text { volubilis } & \text { L. } & \text { en } & \text { los } \\ \text { tratamientos, a diferentes días después de } & \text { siembra. }\end{array}$

\begin{tabular}{|c|c|c|c|c|}
\hline \multirow[b]{2}{*}{ Tratamientos } & \multicolumn{4}{|c|}{ Número de cápsulas } \\
\hline & 112 DDS & 128 DDS & 144 DDS & 168 DDS \\
\hline Control & $2 \mathrm{a}$ & $3 a$ & $8 \mathrm{a}$ & $10 \mathrm{a}$ \\
\hline Micorriza & $3 a$ & $4 \mathrm{a}$ & $10 \mathrm{a}$ & $13 \mathrm{a}$ \\
\hline Lombriabono & $2 \mathrm{a}$ & $3 \mathrm{a}$ & $9 \mathrm{a}$ & $11 \mathrm{a}$ \\
\hline
\end{tabular}

afectan positivamente la formación y desarrollo de capsulas, pues, la relación simbiótica raíz-micorrizas vesículo arbusculares afecta el crecimiento y desarrollo de la planta por otros mecanismos diferentes al mejoramiento de la nutrición del hospedero, mediante la producción de sustancias con actividad hormonal como auxinas, giberalinas $y$ citoquininas (Barea y Azcón-Aguilar, 1983).

Por otra parte, el lombriabono mejora las condiciones fisicoquímicas del suelo en cuanto a aireación, humedad, capacidad de intercambio catiónico, en este sentido, el contenido de humedad en suelo en la época seca en este período de producción no influyó drásticamente, lo que se expresó una buena fructificación, por tanto el número de capsula no fue tan inferior en comparación a la producción de las plantas con micorriza.

Además la producción de cápsulas posiblemente fue influencia por las condiciones ambientales, dado que las temperaturas por encima de 30 ${ }^{\circ} \mathrm{C}$ y los vientos secos producen la desecación del estigma, la inhibición del crecimiento del tubo polínico, y el aborto del embrión, adicionalmente las Iluvias provocan caída del polen, lavado de granos de polen del estigma y pérdida de viabilidad del polen (Rapoport, 2004)

Resultados similares a los obtenidos en este estudio en cuanto al número de capsula fueron obtenido por Melgar et al., (2005) quienes determinaron el efecto de la micorrización
(G. intraradix) en el desarrollo del cultivo de melón (Cucumis melo L.) establecido en un suelo con niveles naturales de fertilidad, reportando que la rendimiento total de fruta fue estadísticamente igual entre los tratamientos, sin embargo, el tratamiento de inoculación con G. intraradix mostro un rendimiento mayor al testigo, mostrando que la inoculación con micorrizas afecta la producción de frutos, debido al efecto de la micorrizas en la asimilación de fósforo por las plantas, pues, el micelio externo y profundamente ramificado además de incrementar el número de sitios para la absorción de $\mathrm{P}$, permite a la raíz explorar un volumen de suelo mayor, captando así fosfatos más allá de la zona de deficiencia.

\section{CONCLUSIONES}

La aplicación de micorrizas vesículo arbusculares comerciales contribuyeron en incrementos en el crecimiento y estimularon la emisión de flores femeninas, flores fecundadas y flores masculinas en las plantas de P. volubilis.

La aplicación de lombriabono a las plantas P. volubilis no es una alternativa eficiente para promover el crecimiento, desarrollo y floración.

\section{AGRADECIMIENTOS}

Los autores expresan sus agradecimientos a la Universidad De Córdoba; por su entera colaboración en el desarrollo de las actividades involucradas en el desarrollo del mismo y $\mathrm{Al}$ 
grupo de investigación GRUBIODEQ que contribuyó en la ejecución de la investigación.

\section{REFERENCIAS}

Acevedo, I. y Pire, R. 2004. Efecto del lombricompost como enmienda de un sustrato para el crecimiento de lechosero (Carica papaya L.). Interciencia 29(5):274 - 279.

AGRONET. 2015. Análisis - Estadísticas. Ministerio de Agricultura y desarrollo Rural, República de Colombia. http:// www.agronet.gov.co [10 de Marzo 2015].

Al-Karaki, G., McMichael, B. y Zak, J. 2004. Field res $\neg$ ponse of wheat to arbuscular mycorrhizal fungi and drought stress. Mycorrhiza 14 (4): 263-269. https:// doi.org/10.1007/s00572-003-0265-2

Antil, R. S, Janssen, B. H. y Lantinga, E. A. 2009. Laboratory and greenhouse assessment of plant availability of organic $\mathrm{N}$ in animal manure. Nutrient Cycling in Agroecosystems 85:95-106. https:// doi.org/10.1007/s10705-009-9251-1

Azcón, C., and Barea, j. 1992. Interactions between mycorrhizal fungi and other rhizosphere microorganisms. In: M.F. A. Chapman and Hall (Ed). Micorrhizal Functioning. An Integrative PlantFungal Process, New York, p 163-198.

Baar, J. 2008. From Production to Application of Ar $\neg$ buscular Mycorrhizal Fungi in Agricultural Systems: Requirements and Needs. Mycorrhiza 4 (1): 361-373. https:// doi.org/10.1007/978-3-540-78826-3_18

Barea, J. y Azcón-Aguilar, C. 1983. Mycorrhiza and their significance on nodulating nitrogen-fixing plants. Advances in Agronomy 36: 1-54. https://doi. org/10.1016/S0065-2113(08)60351-X

Barrera, J., Combatt, E. y Ramírez, Y. 2011. Efecto de abonos orgánicos sobre el crecimiento y producción del plátano Hartón (Musa AAB). Revista Colombiana de Ciencias Hortícolas 5 (2): 186-194.
Barrientos, H. 2014. Análisis de crecimiento funcional, acumulación de biomasa y translocación de materia seca de ocho hortalizas cultivadas en invernadero. Tesis de Maestría, Universidad Mayor de San Andrés, Bolivia

Cabrales, E., Toro, M. y López, D. 2016. Efecto de micorrizas nativas y fósforo en los rendimientos del maíz en Guárico, Venezuela. Temas Agrarios 21(2): 21 - 31. https://doi.org/10.21897/rta.v21i2.898

Cardoso, M. and Kuyper, T. 2006. Mycorrhizas and tropical soil fertility Agriculture. Ecosystems and Environment 116 (1): 72-84. https:// doi.org/10.1016/j.agee.2006.03.011

Catlin, P., and Olsson, E. 1990. Pistillate flower abscission of walnut-Serr, Sunland, Howard and Chandler. HortScience 25:1391-1392

Cruz, E., Sandoval, M., Volke, V., Can, A. y Sánchez, J. 2012. Efecto de mezclas de sustratos y concentración de la solución nutritiva en el crecimiento y rendimiento de tomate. Revista Mexicana de Ciencias Agrícolas 3 (7):1361 - 1373.

De la Rosa-mera, C., Ferrera-Cerrato, R., Alarcón, A., De Jesús Sánchez-Colín, M. y Franco-Ramírez, A. 2012. Aislamiento de consorcios de hongos micorrízicos arbusculares de plantas medicinales y su efecto en el crecimiento de vinca (Catharanthus roseus). Revista Chilena de Historia Natural 85: 187-198. https://doi.org/10.4067/ S $0716-078 \times 2012000200005$

De Prager, M. 2007. Las Endomicorrizas: Expresión bioedáfica de importancia en el trópico. Universidad Nacional de Colombia. Sede Palmira, Palmira, p.352.

Daft, M. J. y Nicolson, T. H. 1966. Effect of endogone mycorrhiza on plant growth. New Phytol 65: 343-350. https:// doi.org/10.1111/j.1469-8137.1966. $\begin{array}{lllllllll}\mathrm{t} & \mathrm{b} & 0 & 6 & 3 & 7 & 0 & \mathrm{x}\end{array}$

Díaz, B., Aguirre, J. y Díaz, V. 2013. Rendimiento de Jatropha curcas L. inoculada con micorriza y aplicación de composta de caña. Revista Mexicana de Ciencias Agrícolas 4 (4): 599-610 
Ekanayake, J., Oyetunji, J., Osonubi, O. and Lyasse, O. 2004. The effects of arbuscular mycorrhizal fungi and water stress on leaf chlorophyll production of cassava (Manihot esculenta Crantz) International Journal of Food, Agriculture and Environment 2 (2): 190-196.

Espinoza, J., Castro, J. y Guillén, R. 2009. Crecimiento de Plukenetia volubilis L. "sacha inchi" cultivado en condiciones de invernadero. Arnaldoa 16 (1): 53-59.

Fanali, C., Dugo, L., Cacciola, F., Beccaria, M., Grasso S., Dachà M., Dugo, P and Mondello, L. 2011. Chemical characterization of Sacha inchi (Plukenetia volubilis L.) oíl. Journal of Agricultural and Food Chemistry 59: 13043-13049. https://doi.org/10.1021/jf203184y

Finck, A. 1988. Fertilizantes y fertilización. Editorial Reᄀverte, México, p 439.

Galindo, P. 2008. Comparación del efecto de inoculación con micorrizas vesículo-arbusculares nativas y comerciales en plantas de frijol (Vigna unguiculata (L.) WALP). Tesis Magíster Scientiarum en Microbiología, Universidad del Zulia, Venezuela.

Gianinazzi, S., Gollotte, A., Binet, M., Van Tuinen, D., Redecker, D., and Wipf, D. 2010. Agroecology: the key role of arbuscular micorrizas in ecosystem services. Mycorrhiza 20:519-530. https:// doi.org/10.1007/s00572-010-0333-3

Herencia, J. F., Ruiz-Porras, J. C., Melero, S. García -Galavis, P. A., Morillo, E. y Maqueda, C. 2007. Comparison between organic and mineral fertilization for soil fertility levels, crop macronutrient concentrations, and yield. Agronomy Journal 99 (4): 973-983. https:// doi.org/10.2134/agronj2006.0168

Karandashov, V. and Bucher, M. 2005 Symbiotic phosphate transport in arbuscular mycorrhizas. TRENDS in Plant Science 10 (1): 22-29. https:// doi.org/10.1016/j.tplants.2004.12.003

Ley, J., Sánchez, J., Ricardo, N. y Collazo, E. 2015. Efecto de cuatro especies de hongos micorrizógenos arbusculares en la producción de frutos de tomate (Solanum lycopersicum L.). Revista Agronomía Costarricense 39(1): 47-59.
Lemus, G. 2005. Control de la caída de flores en nogal 'Serr'. Tierra Adentro 63:18-21.

Luna, J., Romero, I. y Rojas, R. 2016. Hongos micorrizógenos arbusculares y su efecto en el desarrollo de plantas de ají (Capsicum annuum, Solanaceae). Temas Agrarios 21(2): 76 - 85. https:// doi.org/10.21897/rta.v21i2.903

Medina, N., Morejón, R., Cuevas, F. y Díaz, G. 2000. Efecto de la biofertilización con hongos micorri $\neg$ zógenos (MA) en el cultivo del tomate (Lycopersicon esculentum Mill.) Revista Avances de La Habana 2 (4): 1562-3297.

Melgar, J., Dueñas, J y Rivera J. 2005. Factibilidad y efecto de la micorrización en el cultivo de melón establecido en un suelo con niveles naturales de fertilidad. En Martínez, A (Ed). Informe Técnico 2005. Programa de Hortalizas. Fundación hondureña de investigación agrícola, San Pedro Sula.

Montaño, N., Simosa, J. y Perdomo, A. 2009. Respuesta de tres cultivares de berenjena (Solanum melogena L.) a diferentes combinaciones de fertilizante orgánico y fertilizante químico. Revista UDO Agrícola 9(4):807 - 815.

Olivera, R., Ramos, M. y Palacio, S. 2010. Mejoramiento de la producción del frijol (Phaseolus vulgaris L.) con el uso de alternativas de fertilización. Revista Ciencias Holguín 16(2): 1-11.

Palencia, G., Mercado, T. y Combatt, E. 2006. Estudio agroclimático del departamento de Córdoba. Editorial Gráficas el Caribe, Montería. 126 p.

Parodi, G. y Pezzani, F. 2011. Micorrizas arbusculares en dos gramíneas nativas de Uruguay en áreas con y sin pastoreo. Agrociencia 15: $1-10$.

Peña, S. 2008. Protocolo del cultivo de Sacha inchi. Informe final del Subproyecto: Adaptación y validación participativa de paquete tecnológico para la producción competitiva del sacha Inchi (Plukenetia volubilis L.) enlaCuenca del Perené. Centro de Investigación, Educación y Desarrollo (CIED) Selva Central, Junín - Perú, p 86. 
Polito, V., K. Pinney, and J. Labavitch. 1998. Fruit growth and development. In Ramos, D. (ed.) Walnut production manual. Technical editor, California, p139-143

Purakayastha, J. and Chhonkar, P. 2005. Influence of vesicular-arbuscular mycorrhizal fungi (Glomus etunicatum L.) on mobilization of zinc in wetland rice (Oriza sativa L.). Biol Fertil Soils 33 (4): 323- 327. https:// doi.org/10.1007/s003740000330

Rallo, L y J Cuevas. 2004. Fructificación y producción. En: Barranco D, R Fernández -Escobar y L Rallo (Ed). El Cultivo del Olivo. Mundi-Prensa yJunta de Andalucía, Madrid, 159-183.
Rapoport, H. 2004. Botánica y morfología. En: Barranco, D., R FernándezEscobar y L, Rallo (Ed). El Cultivo del Olivo. Mundi-Prensa y Junta de Andalucía, Madrid, p34-60

Suge, J., Omunyin, M. y Omami, N. 2011. Effect of organic and inorganic sources of fertilizer on growth, yield and fruit quality of eggplant (Solanum melongena L).Archives of Applied. Science Research 3 (6):470 - 479.

Yance, M. 2009. Sacha inchi: Moléculas biofuncionales y cosmecéuticas. Editorial CONCYTEC, Lima, p 3. 